\title{
Research and Application of the Beijing Road Traffic Prediction System
}

\author{
Ruimin Li, ${ }^{1}$ Hongliang Ma, ${ }^{1}$ Huapu Lu, ${ }^{1}$ and Min Guo ${ }^{2}$ \\ ${ }^{1}$ Department of Civil Engineering, Tsinghua University, Beijing 100084, China \\ ${ }^{2}$ Beijing Traffic Management Bureau, Beijing 100037, China \\ Correspondence should be addressed to Ruimin Li; lrmin@tsinghua.edu.cn
}

Received 9 November 2013; Accepted 31 December 2013; Published 18 February 2014

Academic Editor: Wuhong Wang

Copyright (c) 2014 Ruimin Li et al. This is an open access article distributed under the Creative Commons Attribution License, which permits unrestricted use, distribution, and reproduction in any medium, provided the original work is properly cited.

\begin{abstract}
As an important part of the urban Advanced Traffic Management Systems (ATMS) and Advanced Traveler Information Systems (ATIS), short-term road traffic prediction system has received special attention in recent decades. The success of ATMS and ATIS technology deployment is heavily dependent on the availability of timely and accurate estimation or prediction of prevailing and emerging traffic conditions. We studied a real-time road traffic prediction system developed for Beijing based on various traffic detection systems. The logical architecture of the system was presented, including raw data level, data processing and calculation level, and application level. Four key function servers were introduced, namely, the database server, calculation server, Geographic Information System (GIS) server, and web application server. The functions, function modules, and the data flow of the proposed traffic prediction system were analyzed, and subsequently prediction models used in this system are described. Finally, the prediction performance of the system in practice was analyzed. The application of the system in Beijing indicated that the proposed and developed system was feasible, robust, and reliable in practice.
\end{abstract}

\section{Introduction}

Along with ever-increasing motorization in China, urban road traffic systems are facing serious congestion issues, especially in the larger cities. The development of Intelligent Transportation Systems (ITS), in particular Advanced Traffic Management System (ATMS) and Advanced Traveler Information System (ATIS), plays an increasingly importation role in urban traffic management. They provide various levels of traffic information and trip advisory to system users, including many ITS information service providers, enabling travelers to make appropriate and informed travel decisions. The success of ATMS and ATIS technology deployment is heavily dependent on timely and accurate estimates of the prevailing and emerging traffic conditions. To implement ATMS and ATIS to meet various traffic control, management, and operation objectives, it is necessary to develop a road traffic prediction system that utilizes advanced traffic prediction models to analyze data, especially real-time traffic data from different sources, to estimate and predict traffic conditions.
In the past few years, real-time traffic prediction systems have been studied and developed in certain cities and regions $[1,2]$, based on simulation or the real-time traffic detection data.

The Traffic Estimation and Prediction system (TrEPS) developed in a dynamic traffic assignment (DTA) research project initiated by the US Federal Highway Administration (FHWA) is a typical traffic prediction system based on simulation. The system is expected to be capable of estimating and predicting traffic information for real-time traffic management and control purposes to meet the information needs in the ITS context $[3,4]$.

Together with IBM, the Singapore Land Transport Authority (LTA) ran a pilot project from December 2006 to April 2007, with a traffic prediction tool based on historical traffic data and real-time feeds with traffic flow conditions from several sources, to predict the levels of congestion up to an hour in advance. The pilot results showed overall prediction results with above $85 \%$ accuracy. Furthermore, 
when more data was available at peak hours, average accuracy reached 90\% [5].

The CAPITALS project was initiated in five European cities (Brussels, Berlin, Paris, Madrid, and Rome) by using and improving existing data resources to establish a platform for information and traffic management services for administration and travelers. A traffic prediction tool was tested and the harmonisation of traffic information in Paris was completed. The five cities above extended their information platforms towards integrated mobility service platforms, in which the prediction tools were developed in Paris, Madrid, and Berlin. In Madrid, estimation of travel times on the M30 motorway ring road was based on a collection of realtime traffic information from the network through detectors and TV cameras and a short-term prediction for congestion analysis. This information was processed in the M30 Traffic Control Centre and communicated via Variable Message Sign (VMS) panels to travelers [6].

As a key element of the Government's Transport 2010 Ten-Year Plan for developing and modernizing the transport system, England's National Traffic Control Centre has gathered real-time information from across the motorway network, improving driving conditions for road users by keeping them better informed and making journey times more reliable. From their website, users obtain the prediction information through the traffic forecaster [7].

The BAYERN ONLINE project launched by the Bavarian State Government in Germany developed the BayernInfo website [8], with one of its main functions providing shortterm, mid-term, and long-term traffic prediction for travelers by using a traffic model called "ASDA-FOTO" [9]. Short-term prediction depends on real-time traffic, midterm prediction depends on traffic events, and long-term prediction depends on traffic demand forecasts. For roads without detectors, the so-called assignment-based methods are applied.

Traffic prediction systems are also under research or construction for some Interstate Highways in America, a case in point being the I-4 Interstate Highway in Orlando, Florida [10]. In addition, most of the developments that have been conducted to date have been carried out in developed countries. In the last decade, many studies have been conducted on short-term traffic flow prediction models and system research in China [11-13], but no practical system has been implemented successfully in the literature to assist real-time traffic operation in cities or highways in China.

To improve traffic management efficiency, the Beijing Traffic Management Bureau (BTMB) launched several ITS systems, including the Beijing Road Traffic Prediction System (BRTPS). In this study we analyzed the development and performance of BRTPS. The system architecture was presented and analyzed in the second section, which is followed with the main functions of BRTPS in the third section. Three key prediction models used in the BRTPS were introduced in the fourth section, as well as the performance analysis in the fifth section. The final section gives a brief conclusion.

\section{System Architecture}

2.1. Logical Architecture. According to system requirements and existing devices and data resources, the logical architecture of the system is shown in Figure 1. The three-level logical architecture includes the following three levels.

2.1.1. Data Resource Level. The data resource level provides the BRTPS system with different data from various existing urban traffic detection systems in Beijing, including the loop detector of the traffic signal control system (covering about three hundred intersections within the second ring expressway), travel time detection system (covering 139 intersections within the fifth ring expressway with vehicle number plate recognition video), microwave traffic flow detection system (covering all expressways in Beijing, with a distance of about $300-800 \mathrm{~m}$ ), probe vehicle detection system (about 20,000 taxi vehicles in Beijing), traffic accident reporting system from the Beijing Traffic Control Center, and other data resources.

2.1.2. Data Processing and Prediction Level. The data processing and prediction level is the core of the BRTPS. It is composed of the following parts.

Data processing module, which provides real-time reliable data for the integrated database via cleaning, coding, and preparation of different data from different sources.

Integrated database, which stores and processes data required by the system, including historical data, real-time processed detection data, prediction data, and statistical analysis results.

Model library, which stores various traffic flow prediction models, traffic accident duration time prediction models, capacity models of intersections and road segments, and analysis models.

Knowledge base, which stores the temporal-spatial relationships produced by traffic flow pattern recognition models and provides basic parameter configuration for the prediction models.

GIS platform, which displays all necessary spatial data and spatial attributes of the system.

The main products of the data processing and prediction level are the predicted values of various traffic flow parameters at different time intervals.

2.1.3. Application Level. The application level is composed of certain application systems supported by the BRTPS, including Personalized Trip Planning and Guiding System and the traffic management system of the traffic control center, and information service providers.

2.2. Physical Architecture. Based on Microsoft. Net Remoting technique, the distributed physical architecture of the system is presented and shown in Figure 2.

The main components of the physical architecture are the four servers, which perform the core functions of the system. 


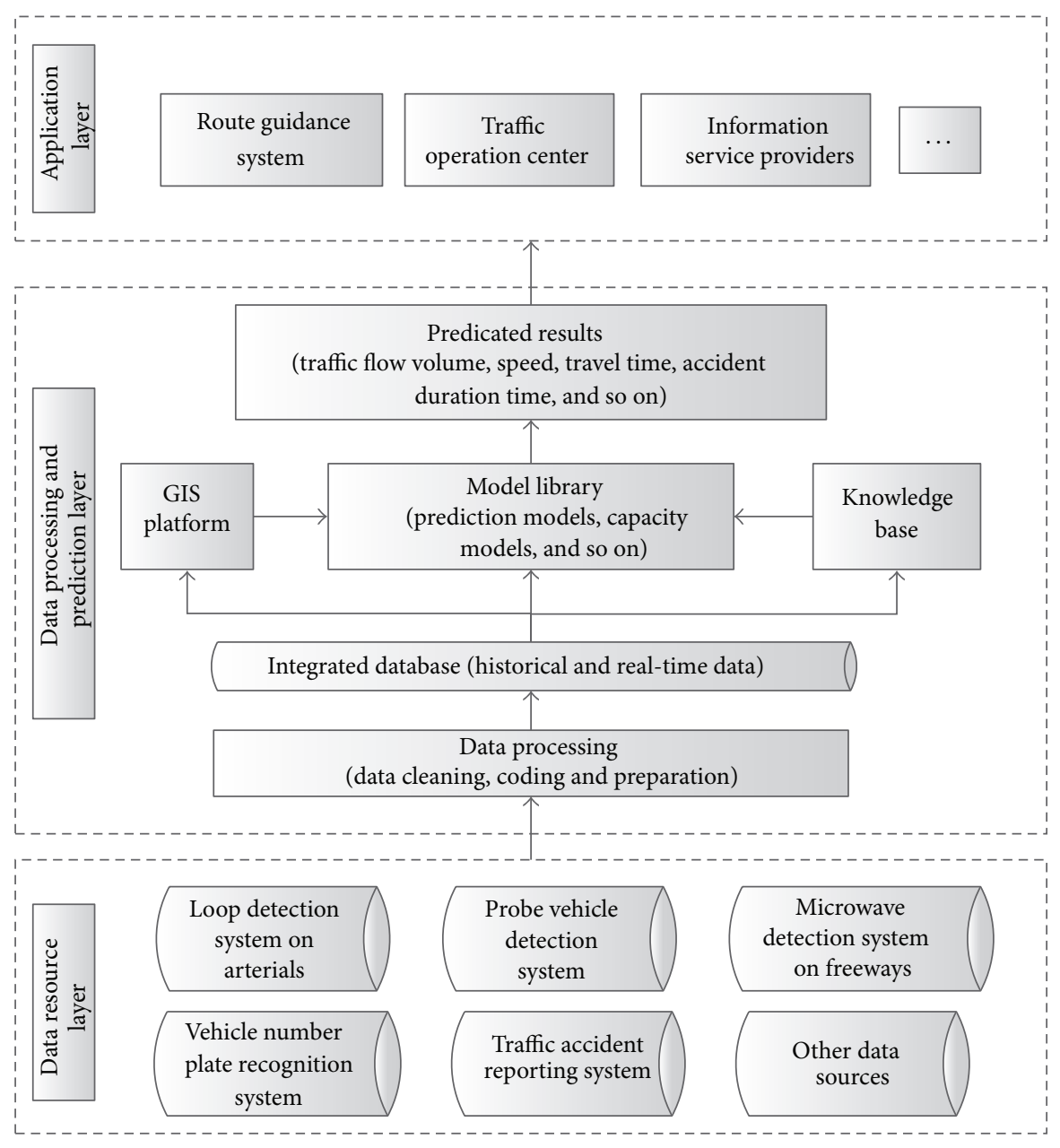

FIgURE 1: Logical architecture of BRTPS.

2.2.1. Database Server. The database server keeps the integrated database running, with the following main functions: (1) obtaining raw data from the existing data center, performing data processing, which transforms the raw data into standardized basic data required by the system, and storing the basic data into the integrated database; (2) storing all necessary basic data and results of traffic flow conditions required by the system; and (3) responding to the requests of reading, writing, and updating traffic flow conditions data from the other three servers.

2.2.2. Calculation Server. The calculation server performs various prediction models used in the system, with the following main functions: (1) obtaining basic data from the database server, calculating traffic flow prediction, road network level of service evaluation, congestion evaluation, incident warning, and temporal-spatial influence analysis based on those data, and then sending the prediction results to the database server; (2) responding to control requests from the web application server by performing requested configuration and thus changing the calculation logic; and (3) responding to the calculation requests from the web application server by performing requested calculations and then sending the results to the web application server.
2.2.3. GIS Server. The main functions of the GIS server include (1) storing urban road network geographical data required by the system; (2) responding to requests of the web application server by analyzing requirements for GIS data and traffic flow data, obtaining the latter from the database server and combining them with GIS data to obtain visualization information, and then sending the visualization information to the web application server; and (3) responding to requests to modify GIS information from the web application server.

2.2.4. Web Application Server. The web application server deals with requests from the other terminals on the network by interpreting requests into requests on GIS data, traffic flow data, and calculation, sending the requests to the other three servers accordingly, and providing user web information based on the information returned from the other servers.

This system provides service via its graphical user interface: system users visit the web application server from their terminals and send requests to the web application server from the browser, which will be analyzed and interpreted by the web application server and sent to the other three servers; these servers will then return the results to the web application server for final processing and displaying on the website for the users. 


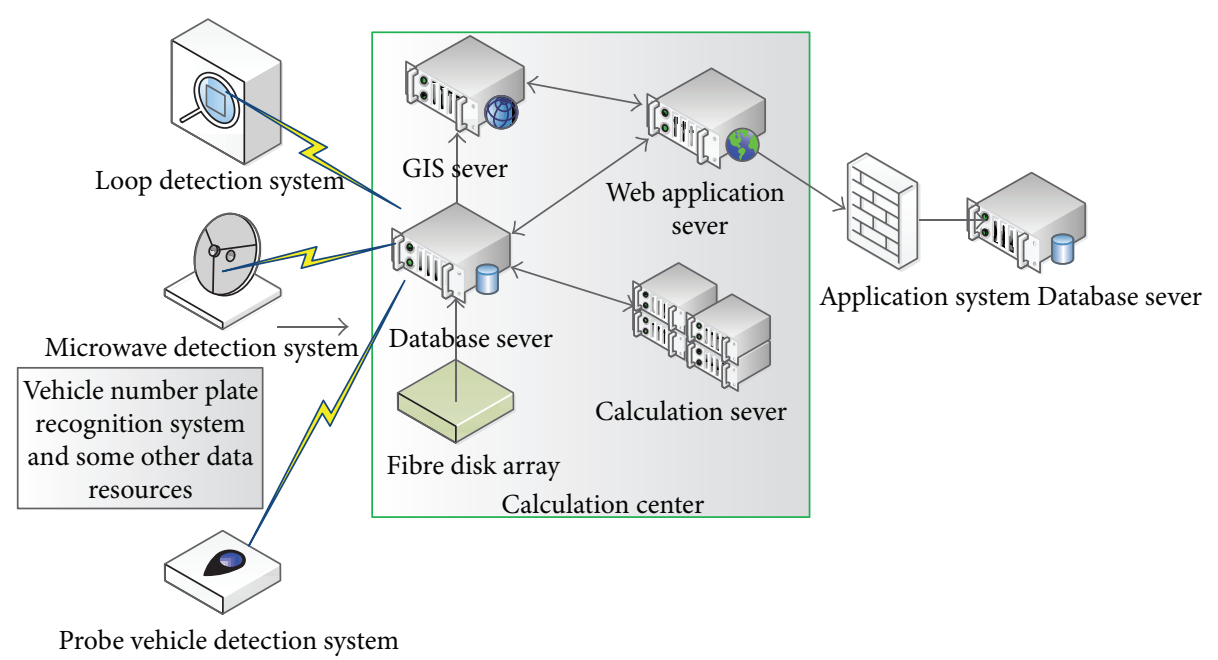

Figure 2: Physical Architecture of the system.

This system also provides service by delivering results for other application systems: based on the requirements of these systems, this system will send the prediction results to these systems at the same time as storing the results in its own integrated database, or other systems obtain the prediction results regularly from the integrated database of this system before performing their own processing and application according to their own needs.

\section{System Functions}

The system mainly consists of the following functions.

Traffic Prediction under Normal Conditions and Prediction Model Update. Based on the integrated database, the system will predict traffic flow conditions with different intervals using various traffic prediction models. Every five minutes, traffic flow parameters, including flow volume, speed, occupancy, and travel time, are predicted with time intervals of 5 mins, 15 mins, 30 mins, $1 \mathrm{~h}$, and $2 \mathrm{~h}$.

Traffic flow prediction models are updated online in accordance with the operation of the system. The correction factors in various prediction models, such as weight factors in the combined prediction model, are continuously adjusted according to the prediction performance or the traffic condition changes to improve prediction accuracy and the model's adaptability to various traffic conditions.

Temporal-Spatial Influence Analysis and Prediction of Traffic Accidents. Based on real-time detected traffic flow data and accident information from the traffic accident reporting system, this system analyzes the temporal-spatial influence of traffic accidents in the Beijing road network. It provides predicted duration time and influence scope of an accident for urban road traffic management administrators.

Traffic Flow Condition Analysis and Evaluation. The system also analyzes and evaluates urban road traffic conditions at the road section, intersection, and region level by adjusting traffic condition evaluation factors and assessing the transport level of service. It also analyzes the detected and predicted data to evaluate the level of traffic congestion.

Urban Road Traffic Changing Trend Analysis. The system can identify the traffic flow changing trend both temporally and spatially, with the immense amount of traffic flow data stored in the system's database. It analyzes the characteristics and trends of traffic flow in different regions, intersections, and sections and the correlation of the traffic flow between them, to provide support for urban road traffic management administrators.

Traffic Information Service. The system can generate traffic flow condition assessment and prediction information, which may be provided for other urban road traffic management systems, organizations, or individuals who have an interest, for example, information service providers. Additionally, it can also disseminate prediction information to public travelers through the VMS or the internet.

\section{Key Prediction Models}

To develop a practical system that can be deployed in the BBTM traffic control center, we presented and modified several models, including the traffic flow parameter correlation model, the capacity calculation model for expressways, urban arterials and intersections, the traffic flow parameter prediction models under normal traffic flow conditions, the Automatic Incident Detection (AID) model, and the accident temporal-spatial influence analysis model [14]. Here we introduce two traffic flow parameter prediction models under normal traffic flow conditions and the accident duration time prediction model.

4.1. Combined Traffic Flow Prediction Model. During the development of the Beijing Road Traffic Prediction System, 


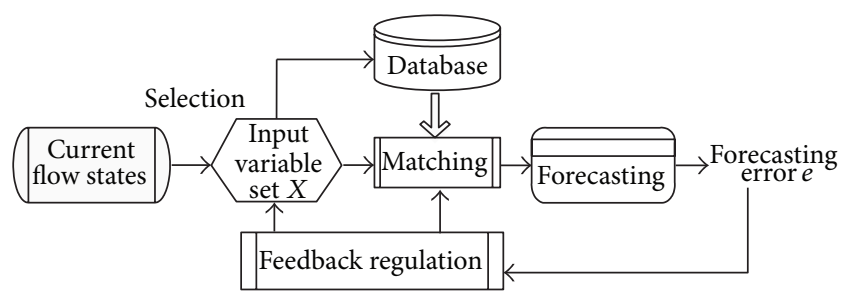

FIGURE 3: Forecasting frame based on NPR.

to find the most suitable prediction model for Beijing's traffic flow conditions, various short-term traffic flow prediction models were proposed for detected and nondetected roads, including the combined traffic flow prediction model [15], the nonparametric regression model [16], and the combined neural network prediction model [17]. The former two models were applied in the system according to the consideration of computation efficiency and prediction accuracy.

The combined prediction model for the BRTPS was considered with the composition of the Discrete Fourier transform model (DFT), Autoregressive model (AR), and Neighborhood Regression model (NR). For convenience, we denoted DFT-AR-NR as the DAN model [15]. Traffic prediction for road sections was not only associated with its historical and recent data of the road section of interest but also with data from adjacent sections. Therefore, a basic form of the DAN model can be represented as [15]

$$
x=\alpha x^{+}+\beta x^{\wedge}+\gamma x^{*}
$$

where $x^{+}, x^{\wedge}$, and $x^{*}$ denote the prediction results of the three submodels, respectively, and $\alpha, \beta$, and $\gamma$ are the weight coefficients of the three submodels, respectively. Adjusting the value of these weight coefficients can strengthen or weaken the role for any of the submodels. The DAN model was mainly used for detected road segments.

4.2. Nonparametric Regression Model. The short-term traffic flow forecasting frame based on nonparametric regression is shown in Figure 3 [16].

The whole system process is as follows.

(1) The system input variable sets were determined by the selection algorithm of current flow states.

(2) The input variable set $X$ was matched among the flow states stored in database to find $K$ optimal matching states. If forecasting time was ample, the linear matching algorithm was the best choice; otherwise we resorted to nonlinear matching algorithm and complex data structure, for example, binary tree and $\mathrm{R}$ tree.

(3) The successfully matching states $Y$ were averaged to obtain the forecasting values.

(4) The forecasting error $e$ was put into the feedback regulation module to adjust the input variable set and matching algorithm.

The nonparametric regression based model was mainly used for nondetected road segments.
4.3. Traffic Accident Duration Time Prediction Model. For traffic accident duration time prediction, a model based on the algorithm of decision tree, Classification and Regression Tree (CART), was presented and applied [18]. The model was developed based on accident records extracted from the accident reporting system of the Beijing Traffic Management Bureau. When an accident occurred, this model will be used to predict the duration of the accident.

\section{System Deployment and Performance Analysis}

5.1. System Deployment. Based on the above models and various data resources, the Beijing Road Traffic Prediction System was developed in the following environment: database system: ORACLE $10 \mathrm{~g}$ database, web server: IIS6, and WebGIS developing and operating system: ArcGIS Server 9.0 from ESRI. The client uses Windows 98 OS or above and web browser IE6.0 or above.

Before the 2008 Olympic Games, the 1.0 vision of BRTPS mainly covered 14 detected expressways and arterial streets within the second ring expressway and was deployed in the traffic control center of BBTM for normal traffic conditions.

In 2011, this system was updated to cover all expressways and arterial streets within the fifth ring expressway, for normal and event traffic conditions. Figure 4 shows the BRTPS interface.

The data used in the system mainly comes from the expressway traffic flow detection system (microwave detectors), travel time detection system based on vehicle number plate recognition, traffic signal control system detectors, and floating car system based on taxi and accident reporting system as mentioned above. It predicts traffic parameters such as flow, speed, and occupancy in $5 \mathrm{~min}, 15 \mathrm{~min}, 30 \mathrm{~min}, 1 \mathrm{~h}$, and $2 \mathrm{~h}$ intervals.

5.2. System Performance Analysis. To understand the prediction performance of the practical system, prediction error analysis was carried out during November 2012.

Fifteen sites selected for the application of the DAN model included ten different expressways in Beijing. Most sites are very congested during morning and evening peak hours. Ten days were selected as test days for all fifteen sites, namely, November 12-16, 2012, and November 26-30, 2012. From 7:00 to $13: 00$ and from 14:00 to 19:00 every day, we selected the detected data and the predicted data hourly. The predicted data included the predicted value of traffic flow, speed, and occupancy in $5 \mathrm{~min}, 15 \mathrm{~min}$, and $30 \mathrm{~min}$ intervals. We mainly analyzed the error performance of speed prediction, which was the most precise among the three traffic flow parameters of volume, speed, and occupancy.

For analysis of system prediction performance, mean absolute percentage error (MAPE) and mean absolute error (MAE) were selected and employed to reflect the accuracy of the predictor. 
TABLE 1: MAPE of speed prediction of the largest error.

\begin{tabular}{|c|c|c|c|c|c|c|c|}
\hline $\begin{array}{l}\text { Site } \\
\text { ID }\end{array}$ & $\begin{array}{c}\text { Prediction } \\
\text { interval (mins) }\end{array}$ & Hour & $\begin{array}{c}\text { Speed } \\
\text { prediction } \\
\text { value }\end{array}$ & $\begin{array}{c}\text { Detected } \\
\text { speed value }\end{array}$ & $\begin{array}{c}\text { Traffic } \\
\text { flow volume }\end{array}$ & $\begin{array}{c}\text { Occupancy } \\
\text { value }(\%)\end{array}$ & $\begin{array}{l}\text { Prediction } \\
\text { error }\end{array}$ \\
\hline 13 & 30 & 17 & 45.91 & 9.83 & 1296 & 83.33 & $367.04 \%$ \\
\hline 13 & 5 & 18 & 39.16 & 8 & 1620 & 66.67 & $389.5 \%$ \\
\hline 10 & 15 & 17 & 63.77 & 12.67 & 4764 & 47.44 & $403.35 \%$ \\
\hline 3 & 5 & 14 & 56.95 & 11.17 & 3348 & 71.17 & $409.85 \%$ \\
\hline 7 & 15 & 9 & 64.73 & 12.67 & 1440 & 52.67 & $410.89 \%$ \\
\hline 10 & 5 & 18 & 59.45 & 11.5 & 3762 & 49.83 & $416.96 \%$ \\
\hline 1 & 15 & 10 & 50.32 & 9.08 & 2742 & 65.5 & $454.17 \%$ \\
\hline 3 & 15 & 18 & 54.71 & 9.17 & 2748 & 70.67 & $496.62 \%$ \\
\hline 7 & 5 & 19 & 58.79 & 9.33 & 438 & 63.33 & $530.12 \%$ \\
\hline 13 & 30 & 17 & 61.21 & 9.08 & 2040 & 66.67 & $574.12 \%$ \\
\hline
\end{tabular}
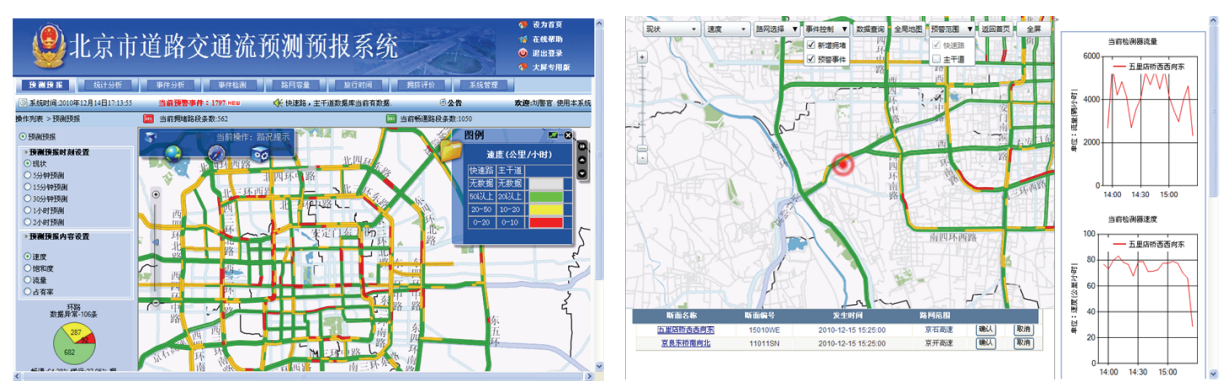

FIGURE 4: System interface.

MAPE and MAE are defined as follows:

$$
\begin{gathered}
\text { MAPE }=\frac{\sum_{t=0}^{N-1}((a b s[V(t+1)-\widehat{V}(t+1)]) /(V(t+1)))}{N}, \\
\text { MAE }=\frac{\sum_{t=0}^{N-1}(a b s[V(t+1)-\widehat{V}(t+1)])}{N},
\end{gathered}
$$

where $V(t+1)$ is the observed traffic flow speed for the time interval $t+1, \widehat{V}(t+1)$ is the predicted traffic flow speed for the time interval $t+1$, and $N$ is the number of intervals for prediction.

The MAPE of speed prediction for different intervals of the fifteen sites over ten days is shown in Figure 5.

From Figure 5, the average MAPE of speed prediction over the ten days increased slowly with increasing prediction interval, specifically by $14.5 \%$ for $5 \mathrm{~min}$ interval, $16.4 \%$ for $15 \mathrm{~min}$, and $16.8 \%$ for $30 \mathrm{~min}$. Eleven sites had MAPE speed prediction at the $5 \mathrm{~min}$ interval below $20 \%$, eleven sites at the $15 \mathrm{~min}$ interval, and ten sites at the $30 \mathrm{~min}$ interval. Thus, speed prediction performance of most selected sites was satisfactory.

The MAPE and MAE of speed prediction at different hours are shown in Figures 6 and 7, respectively. There were no apparent differences in performance in different hours, except for the afternoon peak hours of 17:00 and 18:00. Both MAPE and MAE during afternoon peak hours were larger than that during other hours. The larger errors during the

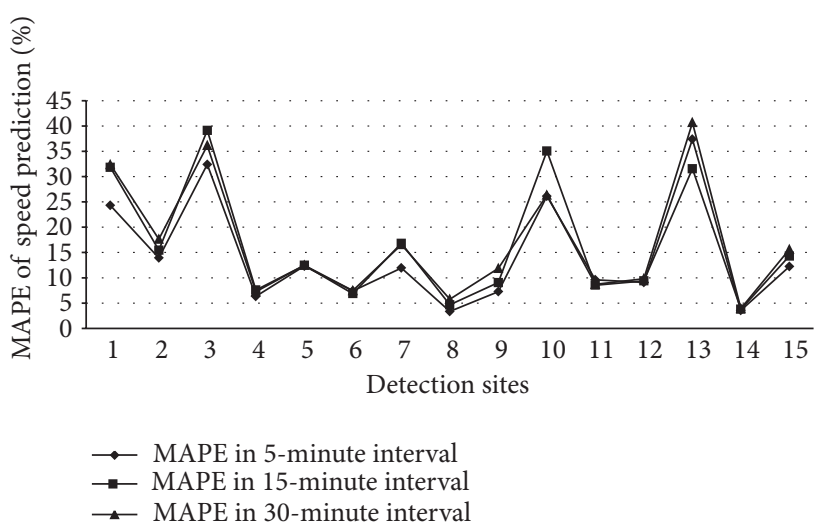

FIGURE 5: MAPE of speed prediction in different intervals of fifteen sites in ten days.

afternoon peak hours indicated that the models deployed in the system may need to be improved for congestion conditions in the future or for some road segments.

The MAPE of speed prediction of the selected sites shows that the accuracy of BRTPS is similar with some other systems, for example, the traffic prediction tool developed by IBM Research for Singapore, in which the overall prediction results were well above the target accuracy of 85 percent [5].

10 speed prediction values with largest prediction error in 5400 data are listed in Table 1, in which the same hour for the same site ID indicates that the hour is in different days. 


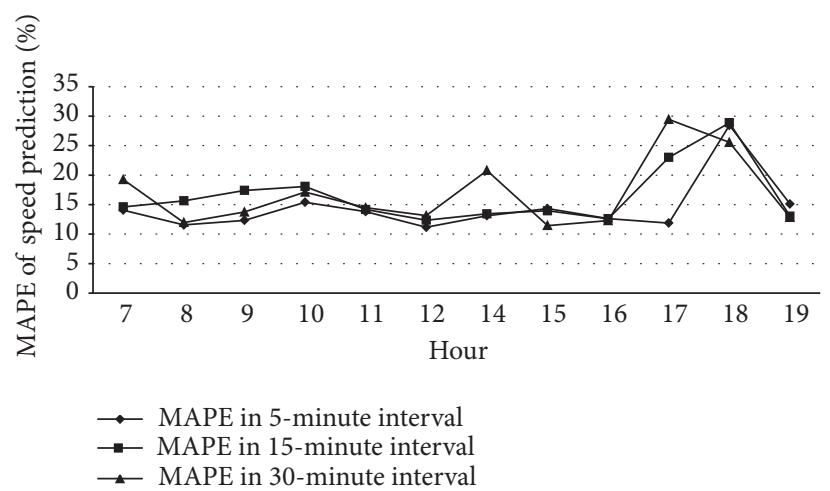

FIGURE 6: MAPE of speed prediction in different hours.

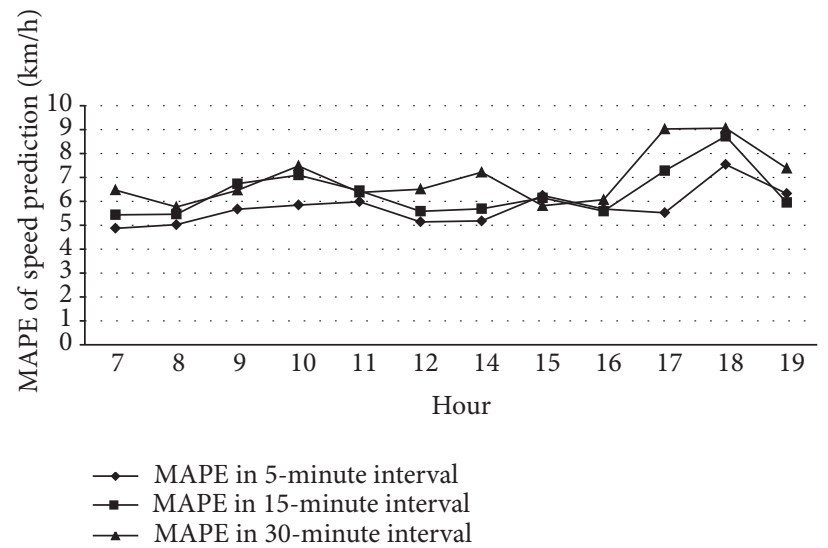

FIGURE 7: MAE of speed prediction in different hours.

From Table 1, we can see that the ten cases with large prediction error almost were undersaturated condition, as shown with the speed and occupancy values. These large speed prediction errors may resulted from two reasons. The first is that the prediction model cannot deal with the traffic condition changing from undersaturated to oversaturated. For example, at 16:45, the traffic condition is free flow and at 17:00 the traffic flow suddenly becomes congested; the combined prediction cannot suit for the changing well. On the other hand, the traffic flow condition at 17:00 may be caused by an event, for example, an accident, and in the current application system did not consider the effect of special event in the prediction model before the event occurred.

\section{Conclusions}

Real-time traffic prediction systems are one of the foundations of ATMS and ATIS. We studied the logic structure, physical structure, and main functions of the Beijing Road Traffic Prediction System deployed in the control center of BTMB. The key prediction models and the online prediction performance were also introduced. Performance analysis indicated that the system satisfied prediction accuracy most of the time for expressways. As discussed, however, during the application period the current system may sometimes produce larger prediction errors, especially during the transition period from free-flow to congested traffic or under congestion conditions. Future prediction accuracy may be improved by refining the developed model based on detected data or by integrating other prediction models based on realtime dynamic traffic assignment.

\section{Conflict of Interests}

The authors declare that there is no conflict of interests regarding the publication of this paper.

\section{Acknowledgments}

This work was supported by Committee of Beijing Science and Technology (Grant no. D070206014007) and National Key Technology R\&D Program (Grant no. 2009BAG13A06).

\section{References}

[1] C. Alecsandru and S. Ishak, "Hybrid model-based and memory-based traffic prediction system," Transportation Research Record, no. 1879, pp. 59-70, 2004. 
[2] B. S. Kerner, H. Rehborn, M. Aleksić, and A. Haug, "Traffic prediction systems in vehicles," in Proceedings of the 8th International IEEE Conference on Intelligent Transportation Systems, pp. 251-256, Vienna, Austria, September 2005.

[3] H. Lieu, A Roadmap for the Research, Development and Deployment of Traffic Estimation and Prediction Systems for RealTime and Off-Line Applications (TrEPS, TrEPS-P), Travel Management Team Office of Operations RD\&T, Federal Highway Administration, 2003.

[4] M. Ben-Akiva, M. Bierlaire, H. Koutsopoulos, and R. Mishalani, "DynaMIT: a simulation-based system for traffic prediction," in Proceedings of the DACCORD Short Term Forecasting Workshop, Delft, The Netherlands, February 1998.

[5] "IBM and Singapore's Land Transport Authority Pilot Innovative Traffic Prediction Tool," IBM Press Release, 2007, http:// www-03.ibm.com/press/us/en/pressrelease/21971.wss.

[6] CAPITALS PLUS TR 4029, 2013, http://cordis.europa.eu/ telematics/tap_transport/research/projects/capitalsplus.html.

[7] Highway Agency, 2013, http://www.highways.gov.uk/trafficinformation/.

[8] BayernInfo, 2012, http://bayerninfo.de/en .

[9] B. S. Kerner, H. Rehborn, M. Aleksic, and A. Haug, "Recognition and tracking of spatial-temporal congested traffic patterns on freeways," Transportation Research Part C, vol. 12, no. 5, pp. 369-400, 2004.

[10] S. Ishak and H. Al-Deek, "Statistical Evaluation of Interstate 4 Traffic Prediction System," Transportation Research Record, no. 1856, pp. 16-24, 2003.

[11] F. You, W. Yu, and H. Rong, "Research on the traffic flow prediction system framework for ITS based on organic computing," in Proceedings of the 10th International Conference of Chinese Transportation Professionals (ICCTP '10), pp. 2180-2191, August 2010.

[12] L. Yong and S. Houbing, "DynaCHINA: a specially-built realtime traffic prediction system for China," in Proceedings of the TRB 86th Annual Meeting Compendium of Papers CD-ROM, Washington, DC, USA, 2007.

[13] Y. Li, X. Hao, and L. Liu, "Short-term traffic flow forecasting based on the improved non-parametric regression," in Proceedings of the 2nd International Conference on Transportation Information and Safety (ICTIS '13), pp. 914-920, 2013.

[14] L. Huapu, S. Yagang, and G. Min, Urban Road Mixed Traffic Flow Analysis Model and Method, China Railway Publishing House, Beijing, China, 2009.

[15] S. Dong, R. Li, L. G. Sun, T. H. Chang, and H. Lu, "Shortterm traffic forecast system of Beijing," Transportation Research Record, no. 2193, pp. 116-123, 2010.

[16] X.-L. Zhang and H.-P. Lu, "The simulation research of nonparametric regression for short-term traffic flow forecasting," in Proceedings of the International Conference on Measuring Technology and Mechatronics Automation (ICMTMA '09), vol. 3, pp. 626-629, Zhangjiajie, China, April 2009.

[17] R. Li and H. Lu, "Combined neural network approach for shortterm urban freeway traffic flow prediction," in Proceedings of the 6 th International Symposium on Neural Network (ISNN '09), vol. 5553 of Lecture Notes in Computer Science, part 3, pp. 1017-1025, May 2009.

[18] R. Li, X. Zhao, X. Yu, J. Li, N. Cheng, and J. Zhang, "Incident duration model on urban freeways using three different algorithms of decision tree," in Proceedings of the International Conference on Intelligent Computation Technology and Automation (ICICTA '10), vol. 2, pp. 526-528, Changsha, China, May 2010. 


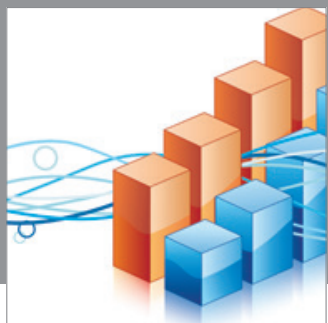

Advances in

Operations Research

mansans

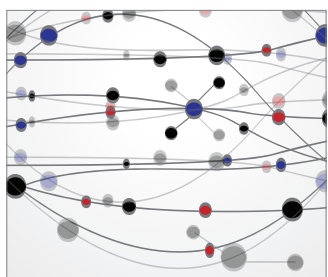

The Scientific World Journal
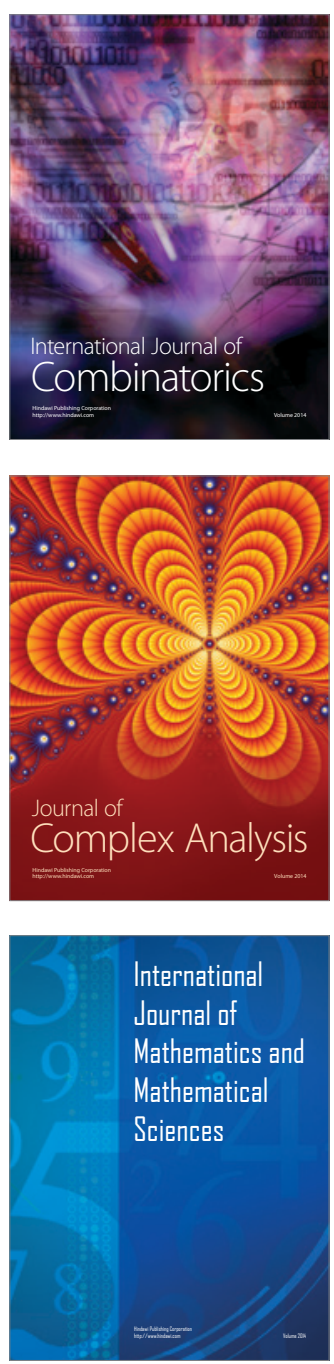
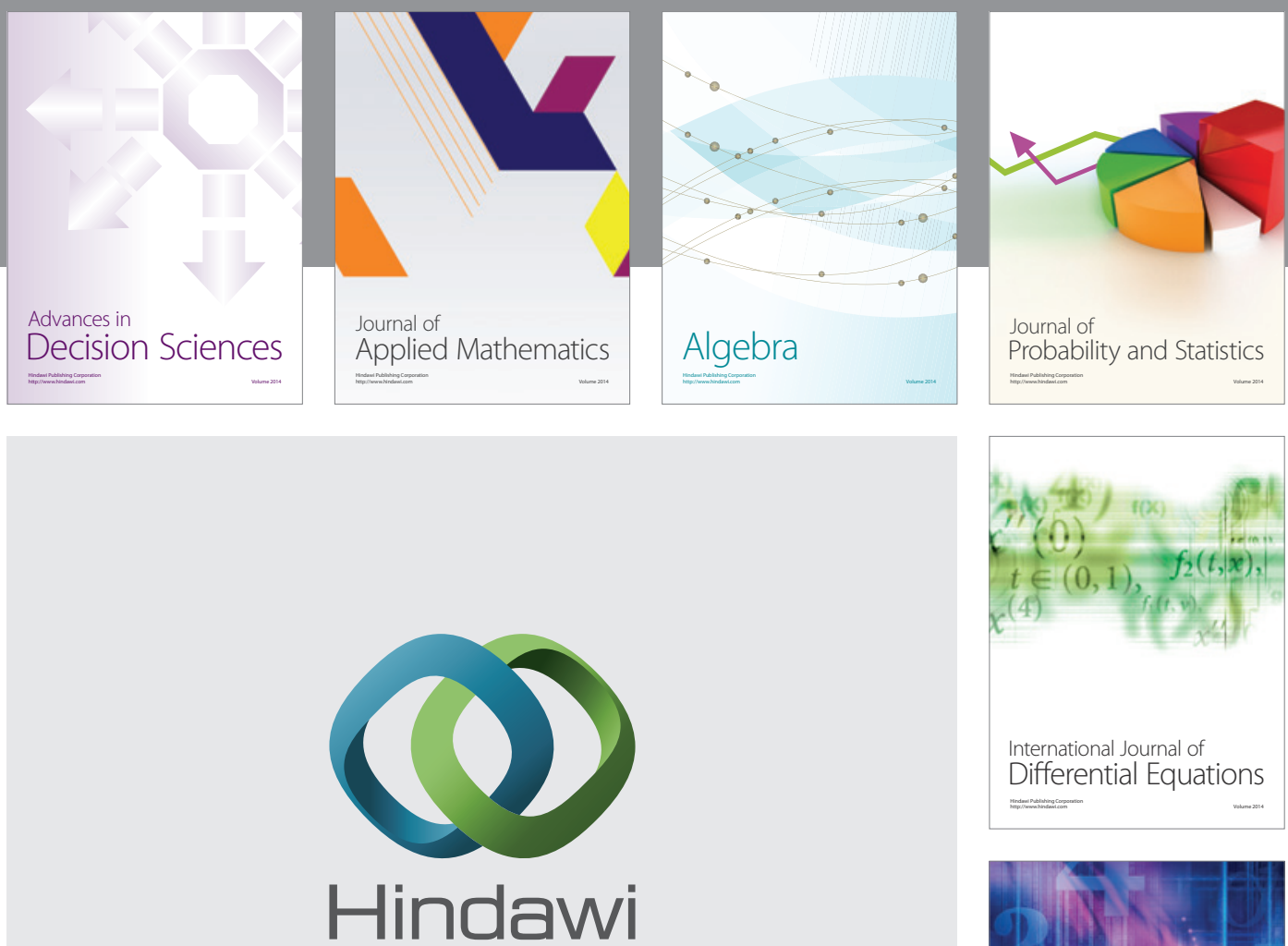

Submit your manuscripts at http://www.hindawi.com
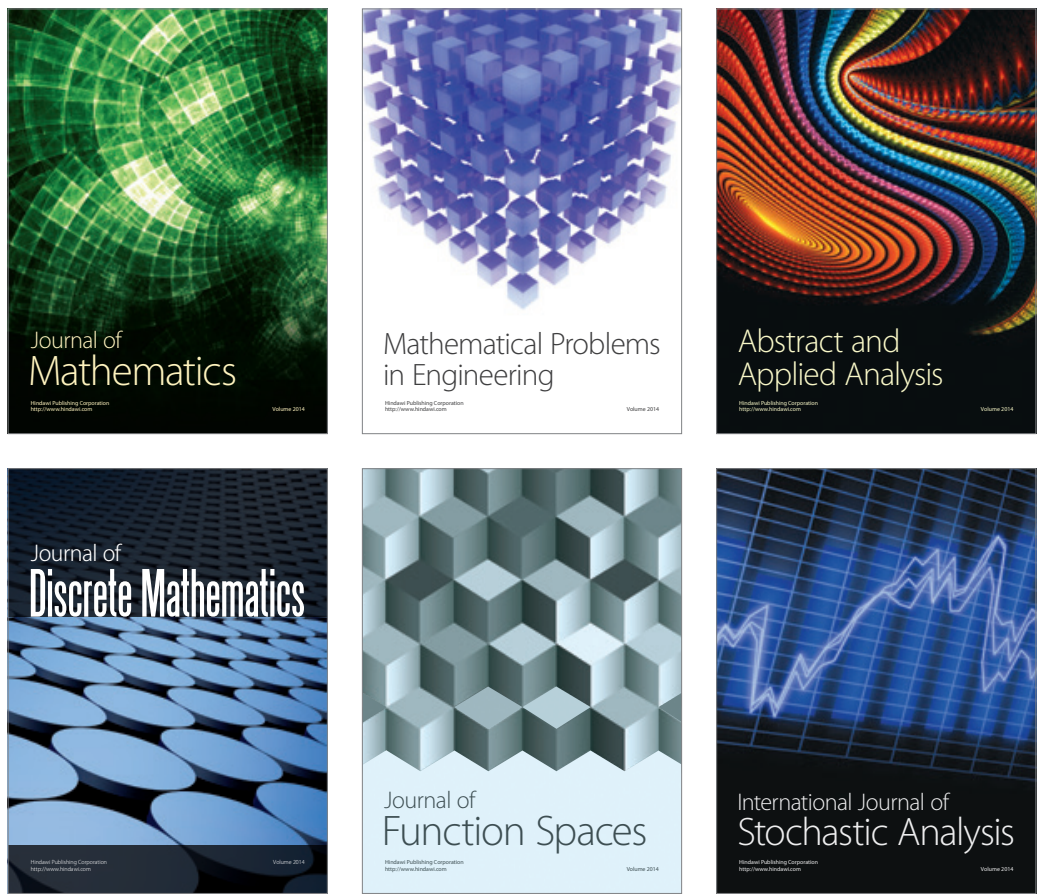

Journal of

Function Spaces

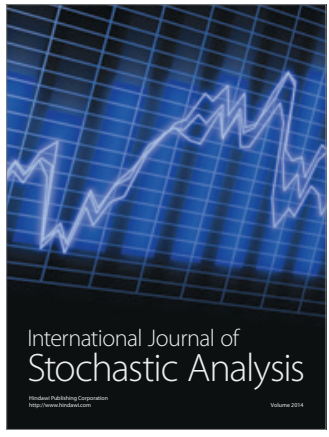

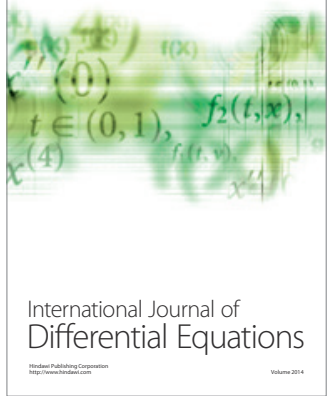
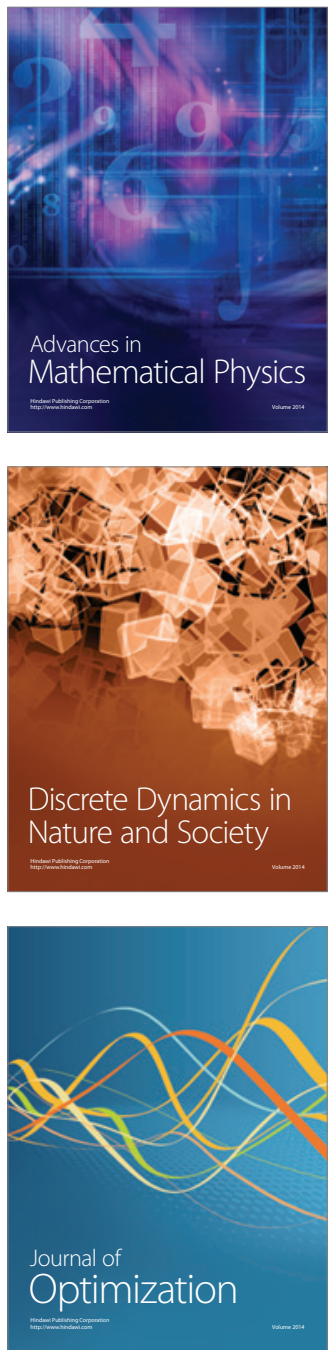